OPEN ACCESS

Edited by:

Jing-Yan Han,

Peking University, China

Reviewed by:

Qiaobing Huang,

Southern Medical University, China

Ruixin Zhu,

Tongji University, China

${ }^{*}$ Correspondence:

Xiaohui Ma

maxiaohui@tasly.com

Feng Yu

yufengcpu@163.com

Specialty section:

This article was submitted to

Vascular Physiology,

a section of the journal

Frontiers in Physiology

Received: 28 November 2017

Accepted: 14 May 2018

Published: 24 September 2018

Citation:

Sun T, Zhang L, Li X, Chen F, Li Y,

Ma $X$ and Yu F (2018) MicroRNA-1

and Circulating Microvesicles Mediate

the Protective Effects of Dantonic

in Acute Myocardial Infarction Rat

Models. Front. Physiol. 9:664.

doi: 10.3389/fphys.2018.00664

\section{MicroRNA-1 and Circulating Microvesicles Mediate the Protective Effects of Dantonic in Acute Myocardial Infarction Rat Models}

\author{
Tingting Sun 1,2, Lihua Zhang ${ }^{2}$, Xinxin $\mathrm{Li}^{2}$, Fengfei Chen ${ }^{1,2}$, Yanchuan $\mathrm{Li}^{2}$, Xiaohui $\mathrm{Ma}^{2 *}$ \\ and Feng $Y_{u^{1 *}}$
}

'School of Basic Medicine and Clinical Pharmacy, China Pharmaceutical University, Nanjing, China, ${ }^{2}$ State Key Laboratory of Core Technology in Innovative Chinese Medicine, Pharmacology and Toxicology Research Centre, Tasly Academy, Tasly Holding Group Co., Ltd., Tianjin, China

Aim: To investigate the protective effect of dantonic in ischemic myocardial damage by evaluating the expression of circulating microvesicles (MVs) and microRNA-1 (miR-1) in two animal models.

Methods: Two animal models of myocardial ischemia were established that were isoproterenol-induced myocardial ischemia (ISO-AMI) rat model and the acute myocardial infarction rat model induced by ligation of the left anterior descending coronary artery (LAD-AMI) of rat. To investigate the protective effect of dantonic, we observed the myocardial infarction size, creatine kinase (CK), lactate dehydrogenase $(\mathrm{LDH})$, aspartate aminotransferase (AST) activities, cardiac troponin I (cTnl) level in serum, and the plasma levels of miR-1 and MVs.

Results: The results showed that pretreatment with dantonic significantly attenuated the LAD-AMI induced myocardial damage by decreasing the size of myocardial infarction, CK, LDH, AST activities, and CTnl level in serum. High dose dantonic treatment could significantly abrogate the increased plasma levels of miR-1 and MVs as compared to the LAD rat model. In addition, pretreatment with dantonic also showed a significant myocardial protective effect through reducing the expression levels of $\mathrm{CK}, \mathrm{LDH}$, and AST as compared to the ISO-AMI model. Whereas the cTnl level was no significant difference between model group and control group, suggesting that the model caused less myocardial damage. In the ISO-induced myocardial ischemia model, there is no significant difference between the model group with the control group of MVs and miR-1 levels. This may be that miR-1 is reported as a biomarker of acute myocardial infarction. The pathological changes of IOS-induced acute myocardial ischemia model are also different from those of acute myocardial infarction.

Conclusion: Dantonic showed the protective effect in these two ischemic myocardial injury rat models, whereas the circulating miR-1 and MVs levels were only ameliorated in the $L A D$ rat model.

Keywords: dantonic, microRNA-1, microvesicles, acute myocardial infarction, protective effects 


\section{INTRODUCTION}

Acute myocardial infarction remains a leading cause of morbidity and mortality in the most developed countries worldwide. It has been estimated that an American suffers from an AMI experience approximately every 42 s (Mozaffarian et al., 2016). According to the Chinese Cardiovascular Disease Report 2016, the number of patients with cardiovascular disease in China is about 290 million, among which the 11 million are with coronary heart disease, acute myocardial infarction mortality rate of 110 people/100,000 people. Various chemical drugs, such as $\beta$-blockers, nitrate preparations, and calcium antagonists, have been used for treatment of AMI. However, long-term use of these chemical drugs might result in significant side effects, such as hypertension and bradycardia (Hlatky et al., 2014). Whereas the significant effects of traditional Chinese medicines (TCM) are becoming more popular (Xin et al., 2013), and they are particularly advantageous for treatment of AMI (Liu et al., 2011).

Dantonic, consisting of Salvia miltiorrhiza (SM), Panax notoginseng (PN), and Borneol, is a widely used TCM for treating ischemic angina pectoris, and has recently finished the phase III clinical trial assessment by the US Food and Drug Administration in 2016, and the results showed Dantonic safe and effective treatment of chronic stable angina. Compared with placebo control group and notoginseng borneol group had statistically significant $(P<0.05)$. The clinical value of Dantonic in the treatment of chronic stable angina pectoris was confirmed again by Phase III clinical trial of FDA. Previous studies showed that Dantonic treatment could significantly reduce the ischemia/reperfusion- (I/R-) and LAD induced myocardial damage and apoptosis, myocardial fibrosis, microcirculatory disturbance (Zhao et al., 2010; Wei et al., 2013; Yang et al., 2013), oxidative stress, and inflammatory response (Horie et al., 2005; Lee et al., 2013; Jun et al., 2014; Ren-an et al., 2014; Chen et al., 2016; Xu et al., 2016).
Many studies focused on the mechanism of AMI by which the circulating microRNAs (miRNAs) takes an active part in the regulation of myocardial infarction (Sala et al., 2014). In AMI, circulating cardiac extracellular vesicles (EVs) contain abundant cardiac-specific miRNAs that serve as indicators of cardiac damage. Among these miRNAs, microRNA-1 (miR-1) is one of the most abundant miRNAs in heart along with a heartand muscle-specific miRNA. Several reports have indicated that miR-1 is upregulated in serum/plasma or urine by AMI and can be used as a diagnostic biomarker of AMI (Ai et al., 2010; Cheng et al., 2010; Kuwabara et al., 2011; Long et al., 2012). Microvesicles (MVs) as a carrier of circulating cell-free miRNAs, is one member of EVs family. Researchers discovered that MVs could be up-regulated by damaged cardiac muscle cells, which reflected an adaptive response to indicate other cells about heart injury. Hence, this present study is aimed to demonstrate the effect of Dantonic on plasma levels of miR-1 and MVs in AMI models.

\section{MATERIALS AND METHODS}

\section{Animals}

Male Sprague-Dawley rats weighing $250 \pm 20$ g obtained from Beijing Vital River Laboratory Animal Technology Co., Ltd. [Beijing, China, Permission No. SCXK (Jing) 2012-0001] were used in this study. Rats were housed in a humidity of $40 \% \pm 5 \%$ and a temperature of $22^{\circ} \mathrm{C} \pm 2{ }^{\circ} \mathrm{C}$ under a $12 / 12 \mathrm{~h}$ light/dark cycle. Rats were free to access water and food, while fasted for $12 \mathrm{~h}$ before the AMI surgery. All animal experiments were approved by the Animal Care and Use Committee of Tasly (TSL-IACUC2016-007).

\section{Agents}

Dantonic was purchased from Tasly Pharmaceutical Co., Ltd. (Tianjin, China). An analysis by high performance
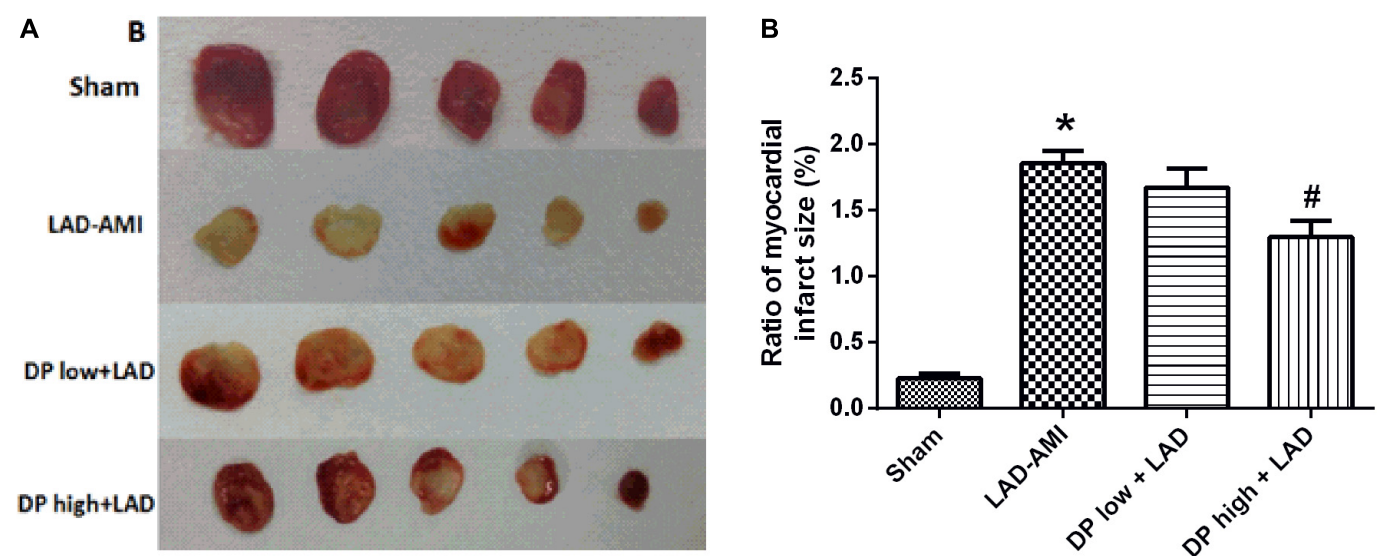

FIGURE 1 | Effects of dantonic on rat myocardial infarct size. (A) Representative slices of ventricle stained by TTC in LAD-AMI model. (B) Quantitative analysis of infarct size-weight ratio: infarct area (white)/whole heart. Sham: control group;0020LAD-AMl: ligate LAD group; dantonic low + LAD: ligate LAD plus pre-treatment with dantonic $167.4 \mathrm{mg} / \mathrm{kg} /$ day group; dantonic high + LAD: ligate LAD plus pre-treatment with dantonic $502.2 \mathrm{mg} / \mathrm{kg} /$ day group. Data are presented as mean \pm SEM. $n=10$ /group ${ }^{*} p<0.05$ vs. Sham group, ${ }^{*} p<0.05$ vs. Model group. 

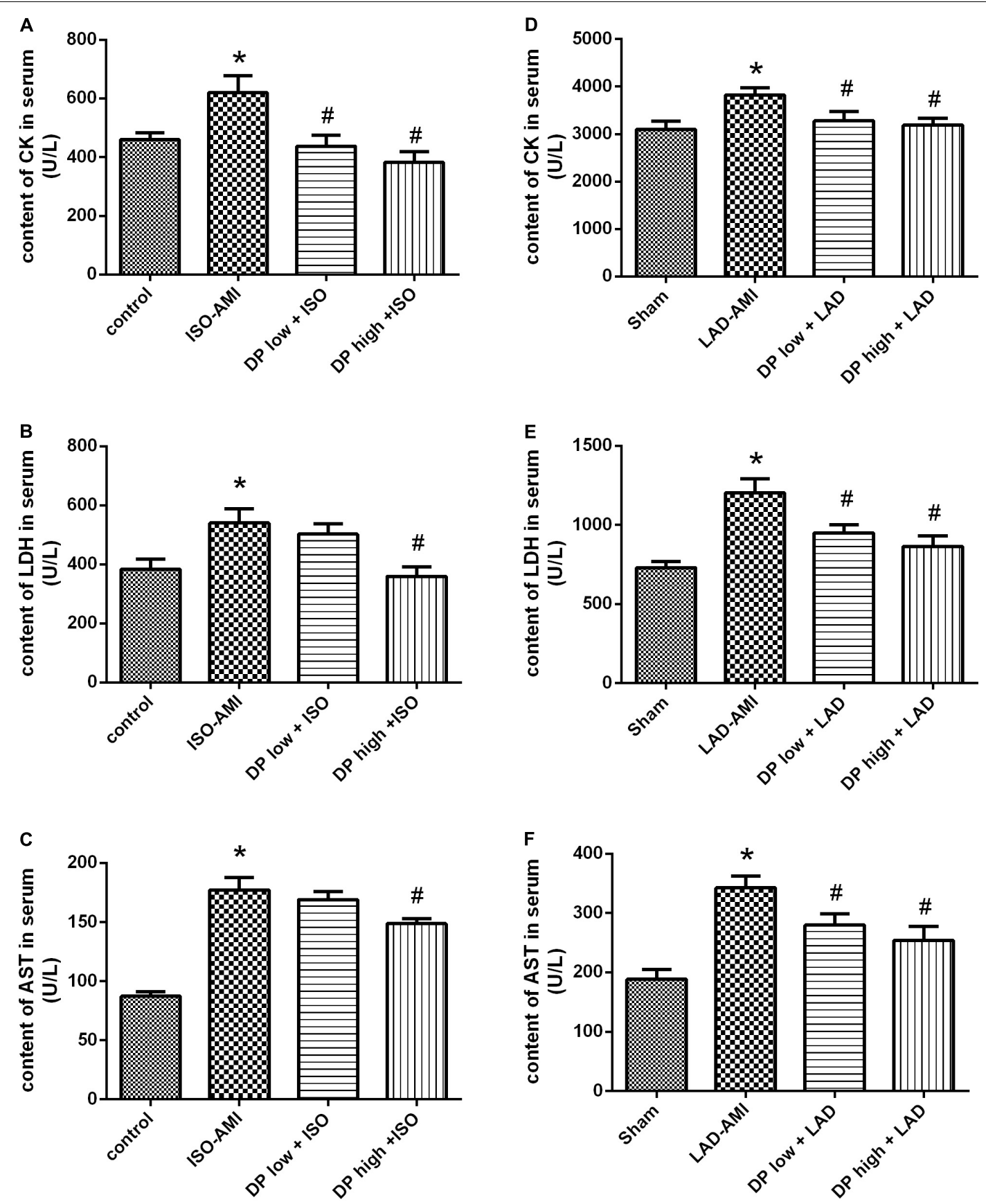

FIGURE 2 | Effects of dantonic on rat serum marker enzymes activities. (A,D) Effect of dantonic on serum creatine kinase (CK) activity against isoproterenol-induced myocardial ischemia (ISO-AMI) and left anterior descending coronary artery (LAD-AMI). (B,E) Effect of dantonic on serum LDH activity against ISO-AMI and LAD-AMI. (C,F) Effect of dantonic on serum AST activity against ISO-AMI and LAD-AMI. Data are presented as mean \pm SEM. ${ }^{*} p<0.05$ vs. sham/control group, $\# p<0.05$ vs. model group.

liquid chromatography was carried out for quality control of Dantonic, with one pill containing $9 \mathrm{mg}$ of SM, $1.76 \mathrm{mg}$ of $\mathrm{PN}, 0.5 \mathrm{mg}$ of Borneol, and $13.74 \mathrm{mg}$ of polyethylene glycol. Isoproterenol was bought from Tokyo Chemical Industry (Tokyo, Japan). Triphenyl tetrazolium chloride (TTC) was bought from Sigma-Aldrich Chemical Co., Ltd. (United States). TTC was dissolved in phosphate buffer at a concentration of $1 \%$. The Elisa determination kit for cardiac troponin I (cTnI) was supplied by the Nanjing Jiancheng Bioengineering Institute (Nanjing, China). MiRNeasy Kit and miRNeasy Spike-In Control were purchased from QIAGEN Co., Ltd. (Germany). RNA PCR Kit and predesigned genespecific primers were purchased from GeneCopoeia Inc. (United States). 

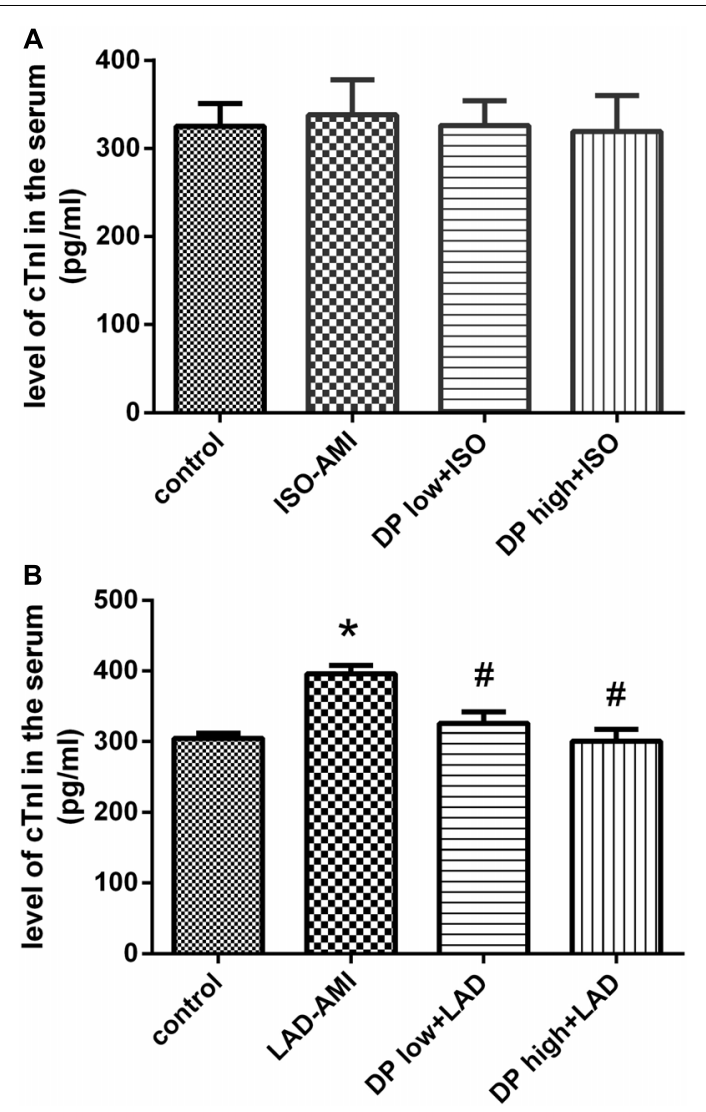

FIGURE 3 | Effects of dantonic on rat serum cTnl activity. (A) Effect of Dantonic on serum cTnl activity against ISO-AMI. (B) Effect of Dantonic on serum cTnl activity against LAD-AMI. Data are represented as mean $\pm \mathrm{SEM}$. ${ }^{*} p<0.05$ vs. Sham/control group, ${ }^{\#} p<0.05$ vs. Model group.

\section{Animal Model}

Two rat models were established in this experiment including isoproterenol-induced myocardial ischemia (ISO-AMI) and the acute myocardial infarction rat model induced by ligation of the left anterior descending coronary artery (LAD-AMI) of rat. Isoproterenol was dissolved in physiological saline and injected subcutaneously to rats $(85 \mathrm{mg} / \mathrm{kg})$ daily for two consecutive days to induce experimental myocardial ischemia (Wang et al., 2009). The control group was given with physiological saline. Rats of LAD-AMI model were anesthetized by an intraperitoneal injection of $20 \%$ urethane prior to surgery. A 6-0 silk suture was placed between the pulmonary and left auricle where the LAD is located. The successful induction of AMI was confirmed based on the observation of the pale color of the anterior portion of the left ventricle. In the sham group, a silk suture was passed through the myocardium without occluding the LAD.

\section{Experimental Protocols}

Rats were randomly divided into four groups in each model including control/sham group, model group (ISO-AMI or LAD$\mathrm{AMI}$ ), dantonic low dose group (dantonic low + ISO or dantonic low + LAD), dantonic high dose group (dantonic high + ISO and dantonic high + LAD). The animals in the treatment groups were pretreated with dantonic by receiving the drug daily by gavage for 5 days. On days 4 and 5, isoproterenol was injected subcutaneously. Dantonic at the dose of $167.4 \mathrm{mg} / \mathrm{kg} / \mathrm{day}$ (low dose group) and $502.2 \mathrm{mg} / \mathrm{kg} / \mathrm{day}$ (high dose group) were respectively onefold and threefold more than the corresponding equivalently effective doses used in clinic. One and a half hour after the last administration of dantonic or water on day 5 , animals were anesthetized and subjected to surgical procedure. In control/sham and model group, rats received the same amount of distilled water in the same way.

\section{TTC Staining}

Blood samples were obtained from abdominal aorta after occluding LAD for $4 \mathrm{~h}$ on day 5 . Hearts were removed and sliced transversely into five sections ( $1 \mathrm{~mm}$ thick) starting from the apex. The slices were incubated with $1 \%$ TTC at $37^{\circ} \mathrm{C}$ for $10 \mathrm{~min}$ under dark conditions to delineate the infarction area. The infarct heart area was represented by unstained (white) color whereas normal heart tissues were stained with red color. Infarction status was expressed as a weigh ratio: infarct area (white)/whole heart.

\section{Biochemical Studies}

Cytosolic enzyme creatine kinase (CK), lactate dehydrogenase $(\mathrm{LDH})$, and aspartate aminotransferase (AST) in serum were measured with autobiochemical analyzer (Hitachi, Ltd., Tokyo, Japan). Serum cTnI activity was detected by enzyme-linked immunoabsorbent assay (ELISA) methods using commercial kits.

\section{MVs Isolation and Flow Cytometric Analysis}

Blood samples were centrifuged at 2,600 $\mathrm{g}$ for $15 \mathrm{~min}$ at room temperature to obtain platelet-poor plasma (PPP), which was then centrifuged at $10,000 \mathrm{~g}$ for $5 \mathrm{~min}$ at room temperature to obtain platelet-free plasma (PFP). PFP was subjected to the ultracentrifugation at $33,000 \mathrm{rpm}$ for $150 \mathrm{~min}$ at $4^{\circ} \mathrm{C}$, to harvest the pelleted MVs, which were then resuspended in $100 \mu 10.9 \%$ sodium chloride and kept at $-20^{\circ} \mathrm{C}$ (Liu et al., 2015).

Dot plots of forward scatter (FSC) vs. side scatter (SSC) showed that $1,2 \mu \mathrm{m}$ standard beads were distributed in the appropriate region by modulating the photomultiplier tube (PMT) voltage. R1 area was gated under the region of $1 \mu \mathrm{m}$ beads which was defined as MVs population. MVs in the R1 gate were further identified by fluorescent (FITC) labeled annexin V.

\section{Quantitative Real-Time RT-PCR Analysis}

Total RNA samples from plasma were prepared using miRNeasy kit according to the manufacturer's instruction. Samples of total small RNAs were reverse-transcribed using the miRNA qRTPCR Detection Kit, and the resulting cDNA was used as a PCR template. The miRNA levels were determined by real-time PCR with the Stratagene Mx3000P Sequence Detector (Agilent, United States) according to the manufacturer's instruction. miR1 primer was designed and verified by GeneCopoeia Inc. The 


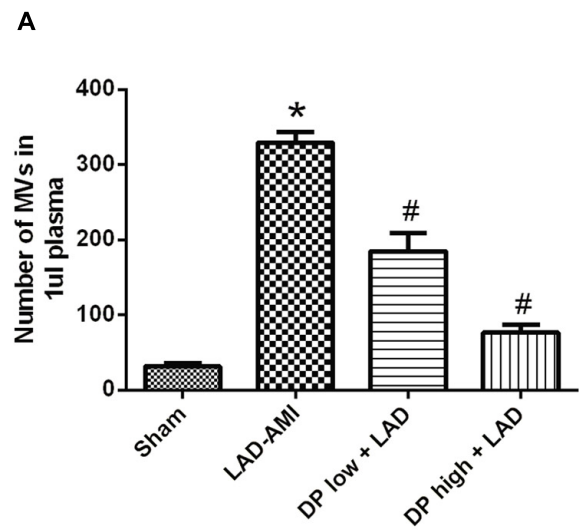

B

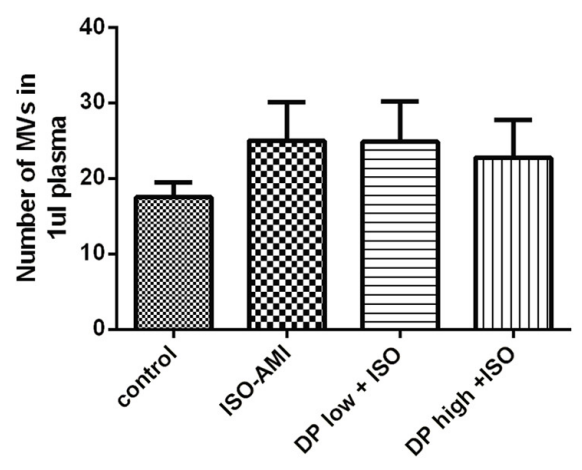

C

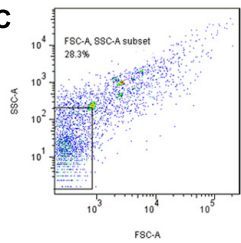

D

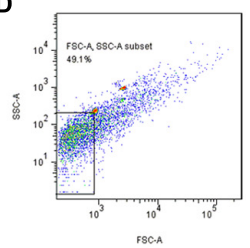

$\mathbf{E}$

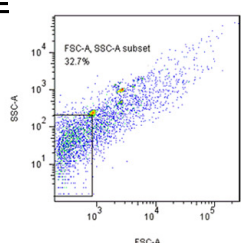

rscen

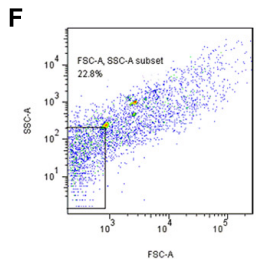

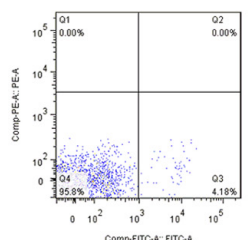
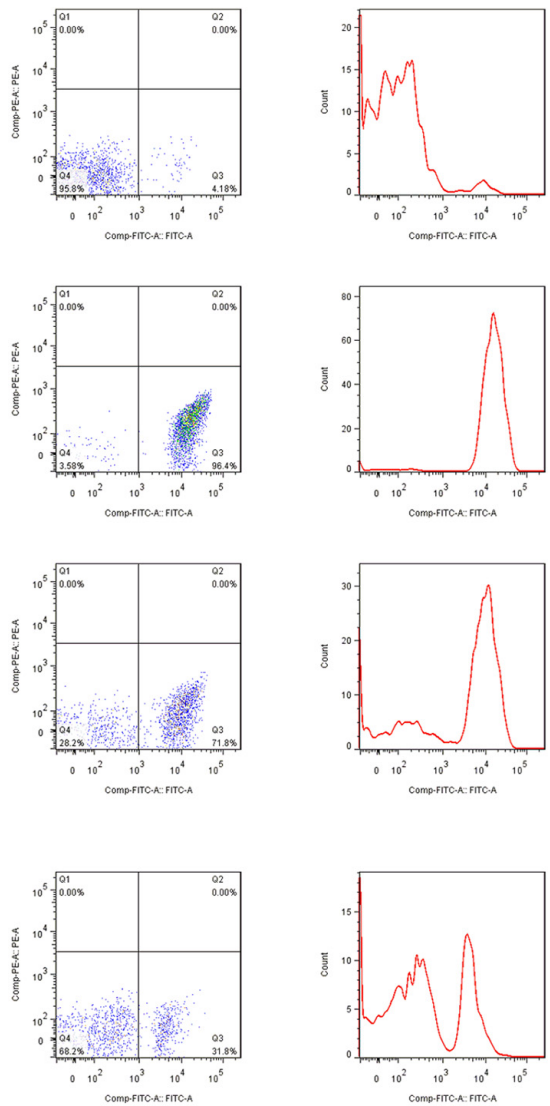

FIGURE 4 | Effects of dantonic on the levels of rat plasma microvesicles (MVs). (A) Effect of dantonic on number of MVs in plasma (events/ $\mu$ I) against LAD-AMI. (B) Effect of dantonic on number of MVs in plasma (events/ $\mu$ I) against ISO-AMI. (C) Representative dot plots and histogram plot in sham group. Representative dot plots of forward scatter (FSC) vs. side scatter (SSC) showed that 1, $2 \mu \mathrm{m}$ latex beads. MVs were identified as events with size less than $1 \mu \mathrm{m}$ within the gate R1. (D) Representative dot plots and histogram plot in LAD-AMl group. (E) Representative dot plots and histogram plot in dantonic low + LAD group. (F) Representative dot plots and histogram plot in dantonic high + LAD group. Data are presented as mean \pm SEM. $n=3 /$ group ${ }^{*} p<0.05$ vs. sham/control group, ${ }^{\#} p<0.05$ vs. model group.

miRNeasy Spike-In Control is a Caenorhabditis elegans miR39 miRNA was amplified as an internal control. All chemical reagents were used in this experiment were analytical grade. The relative miR-1 expression level was calculated using the comparative $\Delta \mathrm{Ct}$ method formula: $2^{-\Delta \Delta C t}$.

\section{Statistical Analysis}

Data were expressed as mean \pm SEM. GraphPad Prism 5.0 program was applied for One-way ANOVA analysis for the statistical comparisons among the groups by using Tukey's test. $P$-values less than 0.05 were considered as statistically significant.

\section{RESULTS}

\section{Dantonic Administration Exerting Beneficial Effects in the LAD-AMI Rat Model}

The infarct weight ratio of each sample was detected to evaluate the cardio protective role of dantonic. Representative heart slices stained by TTC to delineate infarct size are shown in Figure 1A. Apparently, no infarct was observed in myocardial tissue slices from sham group. However, myocardium sections from model group exhibited obvious infarct areas, and administration of dantonic at low and high doses diminishes AMI-induced infarct ratio. Quantitative analysis of the infarct further confirmed that the hearts from dantonic-treated rats showed a significantly smaller infarct weight ratio than those from the model group $(P<0.05$, Figure 1B), suggesting that dantonic administration exerted beneficial effects in LAD-AMI model.

\section{Dantonic Administration Decreasing Serum CK, LDH, and AST Activities}

The activities of the serum marker enzymes CK, LDH, and AST were significantly increased in the ISO-AMI and LADAMI rat models compared to control and sham groups. While in the rats pre-treated with dantonic followed by isoproterenol administration or LAD ligation, the activities of the marker enzymes CK, LDH, and AST in the serum were decreased significantly. $n=10$ /group, $P<0.05$, Figures $2 \mathrm{~A}-\mathbf{F}$. 

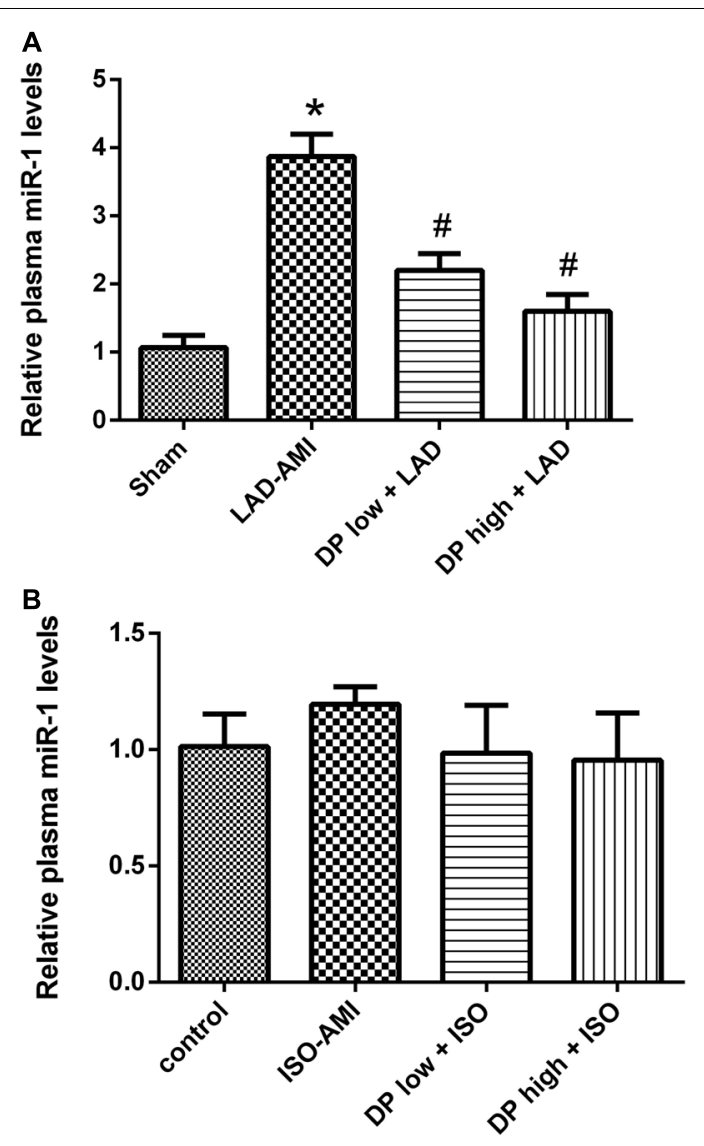

FIGURE 5 | Effect of dantonic on plasma miRNA-1 level. (A) Effect of dantonic on plasma miRNA-1 level induced by LAD-AMI. (B) Effect of Dantonic on the plasma miRNA-1 level induced by ISO-AMI. Data are presented as mean \pm SEM. ${ }^{*} p<0.05$ vs. sham/control group, ${ }^{\#} p<0.05$ vs. model group.

\section{Dantonic Administration Decreasing Serum cTnI Level in LAD-AMI}

The result shows that the level of $\mathrm{cTnI}$ in the serum. Rats induced with LAD showed significant elevation in the levels of cTnI in serum compared to control and sham rats. Pretreatment with dantonic showed a significant decrease in the level of serum cTnI when compared to the LAD-AMI groups. In ISO-AMI the level of serum cTnI has no significant between the control group and model group. ( $n=10$ /group, $P<0.05$, Figure 3 ).

\section{Dantonic Administration Decreasing MVs in the PFP Against LAD-AMI}

Microvesicles were significantly increased in the LAD rat model as compared to the sham group. Dantonic at high dose significantly reduced the plasma levels of MVs as compared to the model group $(P<0.05$, Figure $4 \mathrm{~A})$; But there was no significant difference in the plasma level of MVs between ISOAMI group and control group (Figure 4B). Representative flow cytometry dot plots and histogram plots in LAD model are shown in Figures 4C-F. ( $n=6$ /group).

\section{Dantonic Administration Decreasing the Plasma Level of miRNA-1 Induced by LAD-AMI}

The quantitative Real Time-PCR data demonstrated that ligate LAD could obviously increase the plasma level of miR-1 as compared to sham group, and the miR-1 level was significantly lower in both dantonic-administrated groups compared to LADAMI group $(P<0.05$, Figure 5A). But there was no significant difference in plasma level of miRNA-1 between ISO-AMI group and control group ( $n=3$ /group) (Figure 5B).

\section{DISCUSSION}

Dantonic has been widely applied for prevention and treatment of angina, myocardial ischemia and other cardiovascular diseases in the clinical. More than 450 million people have taken dantonic since it was on the market in 1994. Besides, dantonic has been marketed in many countries (e.g., Canada, Singapore, The United Arab Emirates, Korea, Russia, Cuba, Vietnam, India, and South Africa) as a dietary supplementary for the prevention and treatment of ischemic heart diseases (Chu et al., 2011). Previous studies revealed that the pharmacological mechanism of dantonic against AMI involved anti-platelet aggregation, reducing the overload of calcium and circulating adhesion molecules, ameliorating myocardial fibrosis, protecting against microcirculatory disturbances, inhibiting NADPH oxidase, and modulating the perturbed energy metabolism (Xin et al., 2013; Yang et al., 2013; Zou et al., 2015).

It was discovered that the endogenous plasma miRNAs could be protected in some manner through packaging in microparticles (exosomes, MVs, and apoptotic bodies) or associating with RNA-binding proteins (Argonaute2) or lipoprotein complexes (high-density lipoprotein) to prevent their degradation (Creemers et al., 2012). MVs contain more than 1200 miRNAs and approximately 121 miRNAs can be delivered from one cell to another. Circulating miRNA may be physiologically active and play a pivotal role in cell-to-cell communication as well as MVs. Among these miRNAs, miR1, miR-133a, miR-208, and miR-499 are muscle-enriched which are crucial to regulate the metabolism of the myocardium. Several reports have indicated that miR-1 is upregulated in serum/plasma or urine by AMI and can be used as a biomarker of AMI (Ai et al., 2010; Cheng et al., 2010; Long et al., 2012).

Most of the previous researches about muscle-enriched miRNAs were conducted on patients in clinical trials. But unlike the current study, this time we focused on the changes of miR-1 and MVs in the plasma of rats in the AMI model and dantonic treatment groups. Reports indicated that the circulating miRNAs derived from heart might originate from dead cells after MI and the miRNAs level could reflect the degree of myocardial injury. Additionally miR-1 levels were significantly correlated with the cTnI level in serum (Kuwabara et al., 2011). These are consistent with our experimental results conducted on the LAD-AMI 
model. The results of our study revealed that pretreatment with dantonic significantly attenuated the AMI-induced myocardial damage by decreasing the myocardial infarction size, CK, LDH, AST activities, and the cTnI level in serum. Dantonic at high dose could significantly abrogate the increase in the plasma levels of miR-1 and MVs induced by LAD-AMI.

Subcutaneous injection of isoproterenol can establish the myocardial ischemia animal model. ISO in supramaximal doses induces morphological and functional alterations in heart and then generates highly cytotoxic free radicals through autooxidation of catecholamines, which has been implicated as one of the important causative factors in isoproterenol-induced myocardial damage. It has been reported that auto-oxidation of excess catecholamines results in free radical mediated peroxidation of membrane phospholipids and consequently leads to the permeability changes in the myocardial membrane, intracellular calcium overload and irreversible damage. But miR-1 targets e-NOS, PKCE, HSP60, and regulates muscle cell differentiation, cardiac hypertrophy (Sahoo and Losordo, 2014). In this study, the results show that only CK and LDH levels were significantly increased in the ISO induced myocardial ischemia rat model as compared with the control group, whereas dantoic high dose group could significantly reduce the serum content $(P<0.05)$. Other indicators including cTnI, microRNA, microcapsules were not significantly different between the groups. The serum $\mathrm{CK}$ and $\mathrm{LDH}$ results were consistent with those reported in the literature. The cTnI results of the model group were lower than previous report. Microcapsules and miRNAs have not yet reported on this model, and the cause of this

\section{REFERENCES}

Ai, J., Zhang, R., Li, Y., Pu, J., Lu, Y., Jiao, J., et al. (2010). Circulating microrna-1 as a potential novel biomarker for acute myocardial infarction. Biochem. Biophys. Res. Commun. 391, 73-77. doi: 10.1016/j.bbrc.2009.11.005

Chen, L., Li, X., Li, C., Rong, Y., Xiao, Y., Xu, X., et al. (2016). Chinese herbal cardiotonic pill stabilizes vulnerable plaques in rabbits by decreasing the expression of adhesion molecules. J. Cardiovasc. Pharmacol. 68, 215-222. doi: 10.1097/FJC.0000000000000403

Cheng, Y., Tan, N., Yang, J., Liu, X., Cao, X., He, P., et al. (2010). A translational study of circulating cell-free microRNA-1 in acute myocardial infarction. Clin. Sci (Lond). 119, 87-95. doi: 10.1042/CS20090645

Chu, Y., Zhang, L., Wang, X. Y., Guo, J. H., Guo, Z. X., Ma, X. H., et al. (2011). The effect of Compound Danshen Dripping Pills, a Chinese herb medicine, on the pharmacokinetics and pharmacodynamics of warfarin in rats. J. Ethnopharmacol. 137, 1457-1461. doi: 10.1016/j.jep.2011.08.035

Cocucci, E., Racchetti, G., and Meldolesi, J. (2009). Shedding microvesicles: artefacts no more. Trends Cell Biol. 19, 43-51. doi: 10.1016/j.tcb.2008. 11.003

Connor, D. E., Exner, T., Ma, D. D., and Joseph, J. E. (2010). The majority of circulating platelet-derived microparticles fail to bind annexin $\mathrm{V}$, lack phospholipid-dependent procoagulant activity and demonstrate greater expression of glycoprotein Ib. Thromb. Haemost. 103, 1044-1052. doi: 10.1160/ TH09-09-0644

Creemers, E. E., Tijsen, A. J., and Pinto, Y. M. (2012). Circulating microRNAs: novel biomarkers and extracellular communicators in cardiovascular disease? Circ. Res. 110, 483-495. doi: 10.1161/CIRCRESAHA.111.247452

Hlatky, M. A., Shilane, D., Chang, T. I., Boothroyd, D., and Go, A. S. (2014). Incremental prognostic information from kidney function in patients with new onset coronary heart disease. Am. Heart J. 167, 86-92. doi: 10.1016/j.ahj.2013. 10.006 outcome may be due to the different mechanisms of myocardial injury with the AMI-LAD model.

In the present study, we confirmed that pretreatment with dantonic for 5 days significantly prevented myocardial damage induced by ISO-AMI and LAD-AMI. In addition, we reported for the first time the in vivo data demonstrated that pretreatment with dantonic significantly inhibited LAD-induced increase in miR-1 and MVs levels in plasma. MVs were shed by various cells, especially by platelets, endothelial cells and erythrocytes. MVs appeared to be produced in response to the stimuli (Cocucci et al., 2009) and were defined by their capacity to bind to annexin $\mathrm{V}$, which is an adhesion molecule that specifically interacts with phosphatidylserine (Connor et al., 2010). At present, the form and mechanism of MVs are not very clear in vivo and the effect of dantonic on MVs from particular source needs further research.

\section{AUTHOR CONTRIBUTIONS}

TS is the first author and responses for the entire research. XL, $\mathrm{LZ}, \mathrm{FC}$, and YL are assistants of the research. XM and FY are corresponding authors of the article.

\section{FUNDING}

This research was supported by TCM Standardization Projects of China (Grant No. ZYBZH-C-TJ-55) and Tianjin Science and Technology Plan Projects (Grant No. 16PTSYJC00270).

Horie, Y., Han, J. Y., Mori, S., Konishi, M., Kajihara, M., Kaneko, T., et al. (2005). Herbal cardiotonic pills prevent gut ischemia/reperfusion-induced hepatic microvascular dysfunction in rats fed ethanol chronically. World J. Gastroenterol. 11, 511-515. doi: 10.3748/wjg.v11.i4.511

Jun, Y., Chunju, Y., Qi, A., Liuxia, D., and Guolong, Y. (2014). The effects of Compound Danshen Dripping Pills and human umbilical cord blood mononuclear cell transplant after acute myocardial infarction. Exp. Clin. Transplant. 12, 123-128.

Kuwabara, Y., Ono, K., Horie, T., Nishi, H., Nagao, K., Kinoshita, M., et al. (2011). Increased microRNA-1 and microRNA-133a levels in serum of patients with cardiovascular disease indicate myocardial damage. Circ. Cardiovasc. Genet. 4, 446-454. doi: 10.1161/CIRCGENETICS.110.958975

Lee, K. M., Bang, J. H., Han, J. S., Kim, B. Y., Lee, I. S., Kang, H. W., et al. (2013). Cardiotonic pill attenuates white matter and hippocampal damage via inhibiting microglial activation and downregulating ERK and p38 MAPK signaling in chronic cerebral hypoperfused rat. BMC Complement. Altern. Med. 13:334. doi: 10.1186/1472-6882-13-334

Liu, H. X., Wang, S. R., Lei, Y., and Shang, J. J. (2011). Characteristics and advantages of traditional Chinese medicine in the treatment of acute myocardial infarction. J. Tradit. Chin. Med. 31, 269-272. doi: 10.1016/S0254-6272(12) 60002-8

Liu, M., Wang, Y. L., Shang, M., Wang, Y., Zhang, Q., Wang S. X., et al. (2015). Flow cytometric analysis of circulating microvesicles derived from myocardial Ischemic preconditioning and cardioprotection of Ischemia/reperfusion Injury in rats. Zhongguo. Ying. Yong. Sheng. Li. Xue. Za. Zhi. 31, 524-531. [PubMed PMID: 27215020]

Long, G., Wang, F., Duan, Q., Chen, F., Yang, S., Gong, W., et al. (2012). Human circulating microRNA-1 and microRNA-126 as potential novel indicators for acute myocardial infarction. Int. J. Biol. Sci. 8, 811-818. doi: 10.7150/ijbs.4439

Mozaffarian, D., Benjamin, E. J., Go, A. S., Arnett, D. K., Blaha, M. J., Cushman, M., et al. (2016). Heart disease and stroke statistics-2016 update: a report from 
the American Heart Association. Circulation 133, e38-e360. doi: 10.1161/CIR. 0000000000000350

Ren-an, Q., Juan, L., Chuyuan, L., Wenjuan, F., Chunyan, H., Xuemei, Y., et al. (2014). Study of the protective mechanisms of Compound Danshen Tablet (Fufang Danshen Pian) against myocardial ischemia/reperfusion injury via the Akt-eNOS signaling pathway in rats. J. Ethnopharmacol. 156, 190-198. doi: $10.1016 /$ j.jep.2014.08.023

Sahoo, S., and Losordo, D. W. (2014). Exosomes and cardiac repair after myocardial infarction. Circ. Res. 114, 333-44. doi: 10.1161/CIRCRESAHA.114. 300639

Sala, V., Bergerone, S., Gatti, S., Gallo, S., Ponzetto, A., Ponzetto, C., et al. (2014). MicroRNAs in myocardial ischemia: identifying new targets and tools for treating heart disease. New frontiers for miR-medicine. Cell. Mol. Life Sci. 71, 1439-1452. doi: 10.1007/s00018-013-1504-1500

Wang, S. B., Tian, S., Yang, F., Yang, H. G., Yang, X. Y., and Du, G. H. (2009). Cardioprotective effect of salvianolic acid A on isoproterenol-induced myocardial infarction in rats. Eur. J. Pharmacol. 615, 125-132. doi: 10.1016/j. ejphar.2009.04.061

Wei, X. H., Liu, Y. Y., Li, Q., Yan, L., Hu, B. H., Pan, C. S., et al. (2013). Treatment with cardiotonic pills $\left({ }^{\circledR}\right)$ after ischemia-reperfusion ameliorates myocardial fibrosis in rats. Microcirculation 20, 17-29. doi: 10.1111/micc.12002

Xin, X., Zou, H., Zheng, N., Xu, X., Liu, Y., Wang, X., et al. (2013). Metabonomic strategy to the evaluation of Chinese Medicine Compound Danshen dripping pills interfering myocardial ischemia in rats. Evid. Based. Complement. Alternat. Med. 2013:718305. doi: 10.1155/2013/718305

Xu, M., Hao, H., Jiang, L., Wei, Y., Zhou, F., Sun, J., et al. (2016). Cardiotonic pill reduces myocardial ischemia-reperfusion injury via increasing EET concentrations in rats. Drug. Metab. Dispos. 44, 878-887. doi: 10.1124/dmd.116. 069914
Yang, X. Y., Zhao, N., Liu, Y. Y., Hu, B. H., Sun, K., Chang, X., et al. (2013). Inhibition of NADPH oxidase mediates protective effect of cardiotonic pills against rat heart ischemia/reperfusion injury. Evid. Based. Complement. Alternat. Med. 2013:728020. doi: 10.1155/2013/728020

Zhao, N., Liu, Y. Y., Wang, F., Hu, B. H., Sun, K., Chang, X., et al. (2010). Cardiotonic pills, a compound Chinese medicine, protects ischemiareperfusion-induced microcirculatory disturbance and myocardial damage in rats. Am. J. Physiol. Heart Circ. Physiol. 298, H1166-H1176. doi: 10.1152/ ajpheart.01186.2009

Zou, H. M., Zhang, B., Xu, X. C., Su, J., Sun, Y. N., Xue, S., et al. (2015). Urinary metabolomic strategy to evaluate Compound Danshen Dripping Pills for myocardial ischaemia in rats. J. Pharm. Biomed. Anal. 112, 98-105. doi: 10.1016/j.jpba.2015.04.033

Conflict of Interest Statement: LZ, XL, YL, and XM are employed by Tasly Holding Group Co., Ltd. TS and FC are dual cultured by China Pharmaceutical University and Tasly Holding Group Co., Ltd.

The remaining author declares that the research was conducted in the absence of any commercial or financial relationships that could be construed as a potential conflict of interest.

Copyright (C) 2018 Sun, Zhang, Li, Chen, Li, Ma and Yu. This is an open-access article distributed under the terms of the Creative Commons Attribution License (CC BY). The use, distribution or reproduction in other forums is permitted, provided the original author(s) and the copyright owner(s) are credited and that the original publication in this journal is cited, in accordance with accepted academic practice. No use, distribution or reproduction is permitted which does not comply with these terms. 